\title{
Intensity-Dependent Phase-Matching Effects on Four-Wave Mixing in Optical Fibers
}

\author{
Shuxian Song, Member, IEEE, Member, OSA, Christopher T. Allen, Senior Member, IEEE, \\ Kenneth R. Demarest, Senior Member, IEEE, and Rongqing Hui, Senior Member, IEEE
}

\begin{abstract}
A new phase-matching factor is derived for fourwave mixing (FWM) that includes the effects of self-phase and cross-phase modulation in optical fibers. Theoretical results predict that the wavelength of peak FWM efficiency shifts away from the fiber zero-dispersion wavelength and indicate that the conventional phase-matching factor may induce significant errors in FWM calculations. Experiments are presented to verify the new phase-matching factor and the related theoretical results. The measured results agree well with those predicted by the new phase-matching factor.
\end{abstract}

Index Terms-Four-wave mixing (FWM), optical communications, wavelength-division multiplexing (WDM), nonlinear effects.

\section{INTRODUCTION}

$\mathbf{F}$ OUR-WAVE mixing (FWM) is one of the major limiting factors in wavelength division multiplexing (WDM) optical fiber communication systems that use low dispersion fiber [1] or narrow channel spacing. As a result, estimating FWM efficiency is becoming very important for both the design and evaluation of dense wavelength division multiplexed (DWDM) systems.

A well-known formula used for FWM estimation was originally derived by Hill et al. [2] and was later reformulated to include the phase-matching dependent efficiency by Shibata et al. [3]. This formula, which has been widely used in recent years to evaluate the FWM induced crosstalk in WDM systems [4]-[7], can be written as

$$
P_{i j k}(L)=\frac{\eta}{9} D^{2} \gamma^{2} P_{i} P_{j} P_{k} \exp (-\alpha L)\left\{\frac{[1-\exp (-\alpha L)]^{2}}{\alpha^{2}}\right\}
$$

where $P_{i}, P_{j}, P_{k}$ are the input powers for the signals at frequencies $f_{i}, f_{j}, f_{k}$, respectively, $L$ is the fiber length, $\alpha$ is the fiber attenuation coefficient, and the degeneracy factor $D$ equals to three or six for degenerate and nondegenerate FWM, respectively. The nonlinear coefficient $\gamma$ is given by [8]

$$
\gamma=\frac{2 \pi n_{2}}{\lambda A_{\mathrm{eff}}}
$$

Manuscript received November 23, 1998; revised June 29, 1999. This work was supported by Sprint Corporation, NSF, and KTEC under Grant EPSCoR/ECS 963-2617.

S. Song was with the Information and Telecommunications Technology Laboratory (ITTC), University of Kansas, Lawrence, KS 66045 USA. He is now with CIENA Corporation, Linthicum, MD 21090 USA

C. T. Allen, K. R. Demarest, and R. Hui are with the Information and Telecommunications Technology Laboratory (ITTC), University of Kansas, Lawrence, KS 66045 USA.

Publisher Item Identifier S 0733-8724(99)08931-8. where $A_{\text {eff }}$ is the effective fiber core area, $\lambda$ is the vacuum wavelength, and $n_{2}$ is the fiber nonlinear refractive index, which is related to the nonlinear susceptibility $\chi_{1111}$ by [1]

$$
n_{2}=\frac{48 \pi^{2}}{c n^{2}} \chi_{1111}
$$

where $n$ is the refractive index of the fiber core and $c$ is the vacuum speed of light. Also, $\eta$ is the FWM efficiency, which can be expressed as

$$
\eta=\frac{\alpha^{2}}{\alpha^{2}+\Delta k^{2}}\left\{1+\frac{4 \exp (-\alpha L) \sin ^{2}(\Delta k L / 2)}{[1-\exp (-\alpha L)]^{2}}\right\} .
$$

In this expression, $\Delta k$ is the phase-matching factor, which depends on the fiber dispersion and the channel spacing, and can be expressed as

$$
\Delta k=\frac{2 \pi \lambda_{k}^{2}}{c} \Delta f_{i k} \Delta f_{j k}\left[D_{c}+\frac{\lambda_{k}^{2}}{2 c}\left(\Delta f_{i k}+\Delta f_{j k}\right) \frac{d D_{c}\left(\lambda_{k}\right)}{d \lambda}\right]
$$

where $\Delta f_{m n}=\left|f_{m}-f_{n}\right|,(m, n=i, j, k)$ is the channel spacing (separation), $D_{c}$ is the fiber chromatic dispersion, $d D_{c} / d \lambda$ is the dispersion slope, and $\lambda_{k}$ is the wavelength corresponding to the wave at frequency $f_{k}$. Since $\Delta k$ is not a function of signal powers, we will henceforth call this the linear phase-matching factor.

According to (4) and (5), the FWM efficiency depends on the fiber dispersion, the channel separation, and the fiber length, but not on the transmitted power. However, it is well known that intensity-dependent phase matching plays an important role when power levels are high. This can occur in long-distance fiberoptic communication systems when the inline amplifier spacings are large. Intensity-dependent phasematching for FWM has long been modeled in nonlinear optics in the context of modulation instability and parametric gain [9]-[16], and has used for designing nonlinear optical components [17]-[18].

Even though the mechanism that causes modulation instability and FWM is the same, intensity-dependent phase matching factors derived for parametric gain cannot be applied to (1) for FWM for the following reasons. First, no seed is needed for producing the mixing product in (1), while a seed (usually from noise) is necessary for modulation instability [8]. Second, if there is no fiber loss, the FWM predicated by (1) increases linearly with the fiber length, while in modulation instability, the Stokes and anti-Stokes waves grow exponentially [8], [10]. Third, the wavelengths of the newly generated waves in (1) are 
determined by the pump wavelengths, while the frequencies of Stokes and anti-Stokes waves in modulation instability are determined mainly by the pump power [8]-[11].

The intensity-dependent phase-matching factor used for calculating parametric gain [17], [18] did not include the phase modulation caused by Stokes and anti-Stokes waves. A more general phase-match factor was derived by including the phase modulation contributions from all the waves [10]-[11], but under the assumption of a lossless medium. This assumption will not induce significant errors if the nonlinear medium is a bulk nonlinear material or a very short optical fiber (where the total medium loss or attenuation is small), but is hardly acceptable in optical fiber communication networks, where fiber lengths are hundreds or thousands of kilometers and the fiber loss is a very critical parameter for evaluating fiber nonlinearities. Though a numerical analysis of intensitydependent phase-matching [15] did include the effects of loss, no explicit phase-matching factor was determined. In addition, this study was directed primarily toward predicting modulation instability and parametric gain, not FWM crosstalk using formula (1).

In this paper, we derive a FWM intensity-dependent phasematching factor to be used in the formula (1) that includes the effects of fiber loss. The differences between the FWM efficiencies predicted by the linear phase-matching factor (5) and the phase-matching factors derived for the special case of modulation-instability gain are demonstrated and compared with measured results.

\section{DERIVATION OF THE INTENSITY-DEPENDENT PHASE-MATCHING FACTOR}

We assume that three pump waves at frequencies $\omega_{2}, \omega_{3}$ and $\omega_{4}$ are mixed and generate a new weak wave at frequency $\omega_{1}$ through the FWM process. By including the self-phase modulation (SPM) and cross-phase modulation (XPM) of the pump waves [8], [19], the coupled Schrödinger equations for all four waves can be written as follows:

$$
\begin{aligned}
\frac{d}{d z} A_{1}= & -\frac{1}{2} \alpha A_{1}+2 i \gamma\left(\left|A_{2}\right|^{2}+\left|A_{3}\right|^{2}+\left|A_{4}\right|^{2}\right) A_{1} \\
& +\frac{1}{3} D i \gamma A_{2} A_{3} A_{4}^{*} \exp (i \Delta k z) \\
\frac{d}{d z} A_{2}= & -\frac{1}{2} \alpha A_{2}+i \gamma\left(\left|A_{2}\right|^{2}+2\left|A_{3}\right|^{2}+2\left|A_{4}\right|^{2}\right) A_{2} \\
\frac{d}{d z} A_{3}= & -\frac{1}{2} \alpha A_{3}+i \gamma\left(2\left|A_{2}\right|^{2}+\left|A_{3}\right|^{2}+2\left|A_{4}\right|^{2}\right) A_{3} \\
\frac{d}{d z} A_{4}= & -\frac{1}{2} \alpha A_{4}+i \gamma\left(2\left|A_{2}\right|^{2}+2\left|A_{3}\right|^{2}+\left|A_{4}\right|^{2}\right) A_{4} .
\end{aligned}
$$

In these expressions, $A_{j}(z)$ is the complex, electric-field envelope of the wave at frequency $\omega_{j}$, with propagation number $k_{j}(j=1,2,3,4)$. Also, the phase-matching factor $\Delta k$ is given by

$$
\Delta k=k_{1}+k_{4}-k_{2}-k_{3}
$$

which can also be expressed as (5). In writing (6), the FWMinduced depletion of the pump waves has been neglected, since the newly generated wave power is very weak compared with the pump powers.
From (6b)-(6d), the solutions for the pump envelopes can be obtained as follows:

$$
\begin{aligned}
A_{2}(z)= & A_{2}(0) \exp (-\alpha z / 2) \exp \left[-i \gamma\left(\left|A_{2}(0)\right|^{2}\right.\right. \\
& \left.\left.+2\left|A_{3}(0)\right|^{2}+2\left|A_{4}(0)\right|^{2}\right) \exp (-\alpha z) / \alpha\right] \\
A_{3}(z)= & A_{3}(0) \exp (-\alpha z / 2) \exp \left[-i \gamma\left(2\left|A_{2}(0)\right|^{2}\right.\right. \\
& \left.\left.+\left|A_{3}(0)\right|^{2}+2\left|A_{4}(0)\right|^{2}\right) \exp (-\alpha z) / \alpha\right] \\
A_{4}(z)= & A_{4}(0) \exp (-\alpha z / 2) \exp \left[-i \gamma\left(2\left|A_{2}(0)\right|^{2}\right.\right. \\
& \left.\left.+2\left|A_{3}(0)\right|^{2}+\left|A_{4}(0)\right|^{2}\right) \exp (-\alpha z) / \alpha\right] .
\end{aligned}
$$

Substituting these into (6a), we obtain

$$
\begin{aligned}
\frac{d}{d z} A_{1}= & -\frac{1}{2} \alpha A_{1}+2 i \gamma\left(\left|A_{2}(0)\right|^{2}+\left|A_{3}(0)\right|^{2}\right. \\
& \left.+\left|A_{4}(0)\right|^{2}\right) A_{1} \exp (-\alpha z) \\
& +\frac{1}{3} D i \gamma A_{2}(0) A_{3}(0) A_{4}^{*}(0) \exp \left(-\frac{3}{2} \alpha z\right) \\
& \times \exp \left[-i \gamma\left(\left|A_{2}(0)\right|^{2}+\left|A_{3}(0)\right|^{2}+3\left|A_{4}(0)\right|^{2}\right)\right. \\
& \times \exp (-\alpha z) / \alpha] \exp (i \Delta k z) .
\end{aligned}
$$

Using the transformation

$$
A_{1}(z)=B_{1}(z) \exp (-\alpha z / 2)
$$

we can write (9) as

$$
\begin{aligned}
\frac{d}{d z} B_{1}= & 2 i \gamma\left(P_{2}+P_{3}+P_{4}\right) B_{1} \exp (-\alpha z) \\
& +\frac{1}{3} \operatorname{Di\gamma }\left(P_{2} P_{3} P_{4}\right)^{1 / 2} \exp \left(i \phi_{0}\right) \exp (-\alpha z) \\
& \times \exp \left[-i \gamma\left(P_{2}+P_{3}+3 P_{4}\right) \exp (-\alpha z) / \alpha\right] \\
& \times \exp (i \Delta k z) .
\end{aligned}
$$

Here, $\phi_{0}=\phi_{2}(0)+\phi_{3}(0)-\phi_{4}(0)$, where $\phi_{j}(j=2,3,4)$ are the initial phases for the three pump waves, respectively. Also, $P_{j}=\left|A_{j}(0)\right|^{2}(j=2,3,4)$ are the input powers for three pump waves, respectively.

To represent the fiber attenuation effect on the pump waves, we use the following transformation:

$$
B_{1}(z)=C_{1}(z) \exp \left[-\frac{i \kappa_{1}}{\alpha} \exp (-\alpha z)\right]
$$

where $\kappa_{1}=2 \gamma\left(P_{2}+P_{3}+P_{4}\right)$. Substituting (12) into (11), we obtain the following equation for $C_{1}(z)$ :

$$
\begin{aligned}
\frac{d C_{1}}{d z}= & \frac{1}{3} D i \gamma\left(P_{2} P_{3} P_{4}\right)^{1 / 2} \\
& \times \exp \left[i \phi_{0}-\alpha z+i \Delta k z+\frac{i \kappa_{p}}{\alpha} \exp (-\alpha z)\right]
\end{aligned}
$$

where

$$
\kappa_{p}=\gamma\left(P_{2}+P_{3}-P_{4}\right) .
$$

Integrating (13) over the fiber length, we obtain

$$
C_{1}(L)=\frac{1}{3} D \gamma\left(P_{2} P_{3} P_{4}\right)^{1 / 2} \exp \left(i \phi_{0}\right) I(L)
$$

where $I(L)$ is given by

$$
I(L)=\int_{0}^{L} \exp \left[-\alpha z+i \Delta k z+\frac{i \kappa_{p}}{\alpha} \exp (-\alpha z)\right] d z .
$$


An exact evaluation of (16) requires numerical integration. This is because of the presence of the nonlinear, intensitydependent phase term $\frac{i \kappa_{p}}{\alpha} \exp (-\alpha z)$, which decays exponentially along the fiber. An excellent approximation of $I(L)$ can be obtained by recognizing that this nonlinear phase term has its greatest affect for small values of $z$, since the amplitude of the integrand decreases exponentially with increasing values of $z$. In the range $0<z<L_{\text {eff }}$, the nonlinear phase term is well approximated by the linear expression

$$
\frac{i \kappa_{p}}{\alpha} \exp (-\alpha z) \approx \frac{i \kappa_{p}}{\alpha}\left\{1-\frac{1-\exp \left(-\alpha L_{\mathrm{eff}}\right)}{L_{\mathrm{eff}}} z\right\}
$$

where the fiber effective length $L_{\text {eff }}$ is defined by

$$
L_{\mathrm{eff}}=\frac{1-\exp (-\alpha L)}{\alpha} .
$$

Errors in (17) for $L_{\mathrm{eff}}<z<L$ will have a negligible effect on $I(L)$, since the integrand is small in this range. Using (17) to evaluate (16) results in a closed form expression. Substituting this expression into (15) yields

$$
\begin{aligned}
C_{1}(L)= & \frac{1}{3} D \gamma\left(P_{2} P_{3} P_{4}\right)^{1 / 2} \exp \left(i \phi_{0}\right) \exp \left[\frac{i \gamma}{\alpha}\left(P_{2}+P_{3}-P_{4}\right)\right] \\
& \times\left\{\frac{\exp \left(-\alpha L+i \Delta k^{\prime} L\right)-1}{i \Delta k^{\prime}-\alpha}\right\}
\end{aligned}
$$

where $\Delta k^{\prime}$ is the new, intensity-dependent phase-matching factor, given by

$$
\Delta k^{\prime}=\Delta k-\gamma\left(P_{2}+P_{3}-P_{4}\right)\left\{\frac{1-\exp \left(-\alpha L_{\mathrm{eff}}\right)}{\alpha L_{\mathrm{eff}}}\right\}
$$

where $\Delta k$ is the linear phase-matching factor given in (5).

Substituting (19) into (12) and then substituting the resulting expressions into (10), we finally obtain the generated FWM power

$$
\begin{aligned}
P_{1}(L) & =\left|A_{1}\right|^{2} \\
& =\frac{\eta^{\prime}}{9} D^{2} \gamma^{2} P_{2} P_{3} P_{4} \exp (-\alpha L)\left\{\frac{[1-\exp (-\alpha L)]^{2}}{\alpha^{2}}\right\} .
\end{aligned}
$$

In this expression, $\eta^{\prime}$ is the new FWM efficiency, given by

$$
\eta^{\prime}=\frac{\alpha^{2}}{\alpha^{2}+\left(\Delta k^{\prime}\right)^{2}}\left\{1+\frac{4 \exp (-\alpha L) \sin ^{2}\left(\Delta k^{\prime} L / 2\right)}{[1-\exp (-\alpha L)]^{2}}\right\}
$$

where the new phase-matching factor $\Delta k^{\prime}$ is given in (20). As can be seen (21) is identical to (1), except that phase matching term used in the FWM efficiency term is now $\Delta k^{\prime}$, rather than $\Delta k$. Also, $\Delta k^{\prime}$ and $\Delta k$ become identical when the pump powers are small.

A special case of (20) is the equal transmitted power case, where $P_{2}=P_{3}=P_{4}=P_{0}$, and $P_{0}$ is the channel power. In this case, the phase-matching factor becomes

$$
\Delta k^{\prime}=\Delta k-\gamma P_{0} \frac{1-\exp \left(-\alpha L_{\mathrm{eff}}\right)}{\alpha L_{\mathrm{eff}}} .
$$

This is valid for both degenerate FWM $\left(\omega_{2}=\omega_{3}\right)$ and nondegenerate FWM $\left(\omega_{2} \neq \omega_{3}\right)$. We also note that the phasematching factor changes as the wave propagates along the fiber due to the fiber loss. However, for long fibers $\left(L>L_{\mathrm{eff}}\right)$, we can approximate the effective fiber length by

$$
L_{\mathrm{eff}}=\frac{1-\exp (-\alpha L)}{\alpha} \approx \frac{1}{\alpha}
$$

Then, the phase-matching factor is simplified as

$$
\begin{aligned}
\Delta k^{\prime} & =\Delta k-\gamma\left(P_{2}+P_{3}-P_{4}\right)(1-1 / e) \\
& \approx \Delta k-0.63 \gamma\left(P_{2}+P_{3}-P_{4}\right) .
\end{aligned}
$$

Formulas (20) and (25) can be considered as general forms of the intensity-dependent phase-matching factors discussed earlier in this paper. To enable a direct comparison with the earlier formulations, we now replace $\gamma$ in (25) with $m \gamma$, where $m$ is an integer. Then, we can classify (25) into several cases.

1) One pump $\left(P_{0}\right)$, with or without a weak probe, on a lossless fiber. Here, the probe- and Stokes-wave induced phase modulation and are negligible

$$
\Delta k^{\prime} \approx \Delta k-2 \gamma P_{0}, \quad(m=2)
$$

which corresponds to the factor used in [17], [18].

2) Two pumps $\left(P_{1}=P_{2}=P_{0}\right)$, neglecting Stokes wave induced phase modulation and fiber loss:

$$
\begin{aligned}
& \Delta k^{\prime} \approx \Delta k-2 \gamma P_{0}, \quad(\text { Degenerate FWM }, m=2) \\
& \Delta k^{\prime} \approx \Delta k-\gamma P_{0}, \quad(\text { Nondegenerate FWM }, m=1)
\end{aligned}
$$

which corresponds to the factors used in [10], [11].

3) Three pumps $\left(P_{1}=P_{2}=P_{3}=P_{0}\right)$, nondegenerate FWM, neglecting Stokes-wave induced phase modulation and fiber loss:

$$
\Delta k^{\prime} \approx \Delta k-\gamma P_{0}, \quad(m=1)
$$

which corresponds to the factor in [10].

4) Two or three pumps, degenerate or nondegenerate FWM, neglecting all the nonlinear phase modulation contributions:

$$
\Delta k^{\prime} \approx \Delta k, \quad(m=0)
$$

which is simply the linear phase factor (5) as used in [4]-[7].

5) Two or three pumps $\left(P_{1}=P_{2}=P_{3}=P_{0}\right)$, degenerate or nondegenerate FWM, including fiber loss and all pump-induced phase modulation contributions:

$$
\Delta k^{\prime} \approx \Delta k-0.63 \gamma P_{0}, \quad(m=0.63)
$$

which is new factor derived in this paper, (20). Note that this phase-matching factor accounts for the continuous change of intensity-induced phase-matching contributions along the fiber length, where the localized values of $m$ evolve from 1 at the fiber input to 0 when $z \gg L_{\text {eff }}$. 


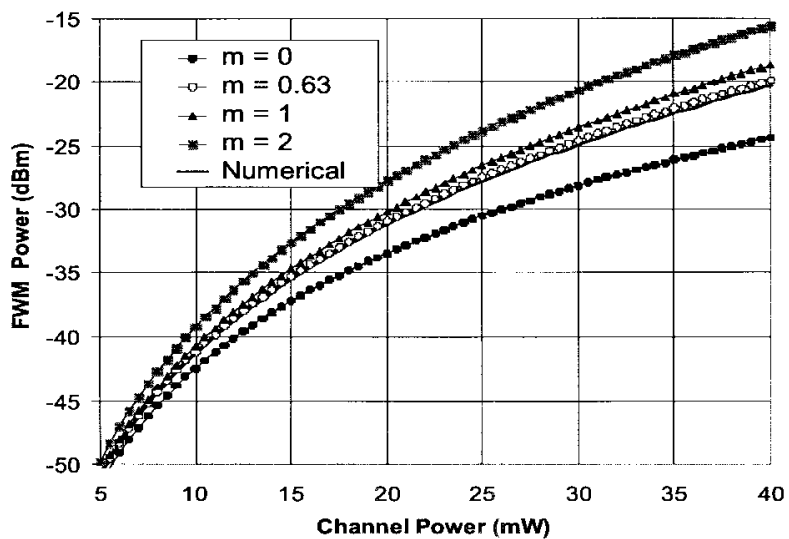

Fig. 1. FWM power as a function of the channel power. Fiber length: $17.5 \mathrm{~km}$, loss: $0.25 \mathrm{~dB} / \mathrm{km}$, dispersion: $0.5 \mathrm{ps} / \mathrm{km}-\mathrm{nm}$, dispersion slope: 0.08 ps $/ \mathrm{km}-\mathrm{nm}$, fiber effective core area: $50 \mu \mathrm{m}^{2}$, nonlinear refractive index: 2.68 $\times 10^{-20} \mathrm{~m}^{2} / \mathrm{W}$, channel spacing: $0.8 \mathrm{~nm}$.

Clearly, different $m$ values correspond to different circumstances and approximations. In the next two sections, we compare the FWM powers predicted using these factors, along with "exact" calculations and measured results.

\section{COMPARISON OF ANALYTICAL FORMULAS}

First, consider the FWM power generated at the end of $17.5 \mathrm{~km}$ of dispersion-shifted fiber when two pump frequencies are present (i.e., the degenerate case), each with channel power $P_{0}$. Fig. 1 shows the calculated FWM powers vs. $P_{0}$ using four formulas. The solid curve is obtained using numerical integration of (16). We will consider this to be the "exact" result. Other curves correspond to phase-matching factors with different $m$ values, including the new phasematching factor (20) (i.e., $m=0.63$ ). For these calculations, the fiber loss is $0.25 \mathrm{~dB} / \mathrm{km}$, the fiber effective core area is 50 $\mu \mathrm{m}^{2}$, the fiber nonlinear refractive index is $n_{2}=2.68 \times 10^{-20}$ $\mathrm{m}^{2} / \mathrm{W}$, the fiber dispersion is $0.5 \mathrm{ps} / \mathrm{km}-\mathrm{nm}$ at $1558 \mathrm{~nm}$, and the dispersion-slope is $0.08 \mathrm{ps} / \mathrm{km}-\mathrm{nm}^{2}$. The pump wavelengths are $1558 \mathrm{~nm}$ and $1558.8 \mathrm{~nm}$, resulting in a channel spacing of $0.8 \mathrm{~nm}$. As can be seen, all four formulas agree well for channel powers below $5 \mathrm{~mW}(7 \mathrm{dBm})$, where the effects of SPM and XPM on the FWM power are negligible. However, as the channel power is increased, these effects become significant. At a channel power of $40 \mathrm{~mW}(16 \mathrm{dBm})$, use of the linear phase-matching factor $(m=0)$ yields roughly a $4 \mathrm{~dB}$ error in the FWM power, whereas neglecting the fiber loss $(m=1)$ results an error of $1.6 \mathrm{~dB}$. This is in contract to calculations obtained using the new FWM phase matching factor $(m=$ 0.63 ), which results in an error of only about $0.5 \mathrm{~dB}$.

Next, we consider the FWM power generated when the pump powers are fixed at $40 \mathrm{~mW}(16 \mathrm{dBm})$ each and the channel spacing is varied. Fig. 2 shows the FMW powers predicted by the same three analytic formulas used above for the case where the fiber has length $20 \mathrm{~km}$, the dispersion is $0.4 \mathrm{ps} / \mathrm{km}-\mathrm{nm}$ at $1556 \mathrm{~nm}$, the dispersion slope is $0.08 \mathrm{ps} / \mathrm{km}$ $\mathrm{nm}^{2}$, and the fiber nonlinear refractive index and effective area are the same as in the previous example. One channel is located at $1556 \mathrm{~nm}$ and the other channel's wavelength is

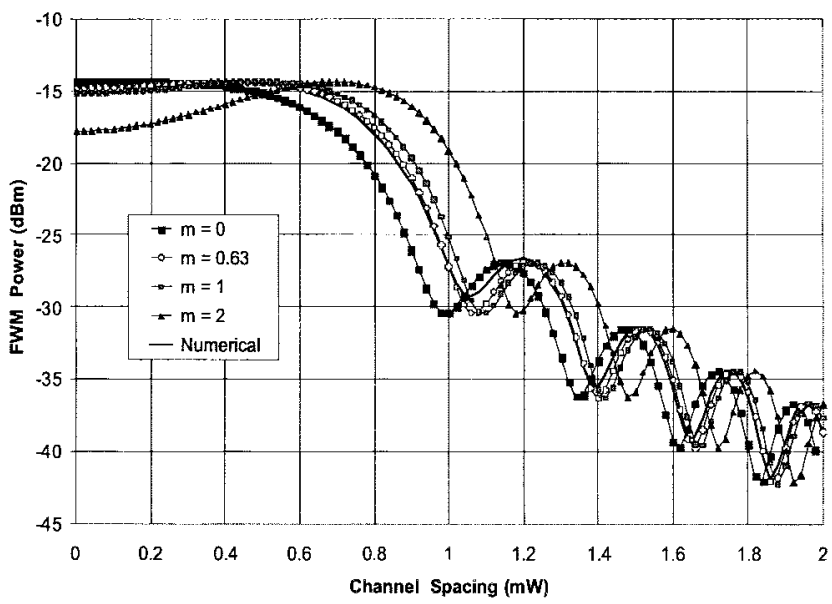

Fig. 2. FWM power as a function of the channel spacing. Fiber length: $20 \mathrm{~km}$, loss: $0.25 \mathrm{~dB} / \mathrm{km}$, dispersion: $0.4 \mathrm{ps} / \mathrm{km}-\mathrm{nm}$, dispersion slope: 0.08 $\mathrm{ps} / \mathrm{km}-\mathrm{nm}$, fiber effective core area: $50 \mu \mathrm{m}^{2}$, nonlinear refractive index: 2.68 $\times 10^{-20} \mathrm{~m}^{2} / \mathrm{W}$, channel spacing: $0.8 \mathrm{~nm}$, channel power: $40 \mathrm{~mW}(16 \mathrm{dBm})$.

varied. As can be seen from the solid ("exact") curve, SPM and XPM shift the maximum FWM point away from the zero channel-spacing point. The amount of shift depends on the channel power. In this case, it is about $0.5 \mathrm{~nm}$. The new phasematching factor $(m=0.63)$ provides excellent agreement with the exact result. The linear phase-matching factor $(m=0)$ suffers from significant errors (up to $5 \mathrm{~dB}$ ) and does not predict correct wavelength separation for maximum FWM production. Although neglecting fiber loss $(m=1)$ caused about $2.2 \mathrm{~dB}$ error, which is significanly better than either totally neglecting intensity-induced phase-modulation $(m=0)$ or the case of parametric gain $(m=2)$, it was still more than $1 \mathrm{~dB}$ worse than the new formula derived here.

\section{EXPERIMENTAL VERIFICATION}

Experiments were conducted to measure the FWM efficiency in a 17.5-km section of dispersion-shifted fiber. The FWM power was measured while the pump wavelengths were varied in unison. In this way, the wavelength spacing was fixed, but the fiber dispersion was different for each measurement. Two $\mathrm{CW}$ tunable lasers were used, each with a polarization controller to align the polarization states. The open circles in Fig. 3(a) are the measured FWM efficiency as a function of the lowest pump wavelength, where the FWM efficiency is defined as the FWM power, normalized to its maximum. The pump powers for this case were each $8 \mathrm{~mW}(9 \mathrm{dBm})$ and the channel spacing was $1 \mathrm{~nm}$. The fiber had an attenuation of $0.5 \mathrm{~dB} / \mathrm{km}$, an effective core of $50 \mu^{2}$, a zero dispersion wavelength of $1551 \mathrm{~nm}$, and a dispersion slope of $0.075 \mathrm{ps} / \mathrm{km}-\mathrm{nm}$. Also shown in this figure are the calculated FWM efficiencies using the new FWM phase-matching formula $(m=0.63)$ and the old FWM phasematching formulas $(m=0, m=1$, and $m=2)$. As can be seen, all four formulas agree well with the measured results. This should be expected, since the pump powers were low enough so that the effects of SPM and XPM could be neglected. For this case, the peak of the FWM efficiency is close to the zero dispersion wavelength $(1551 \mathrm{~nm})$, and 


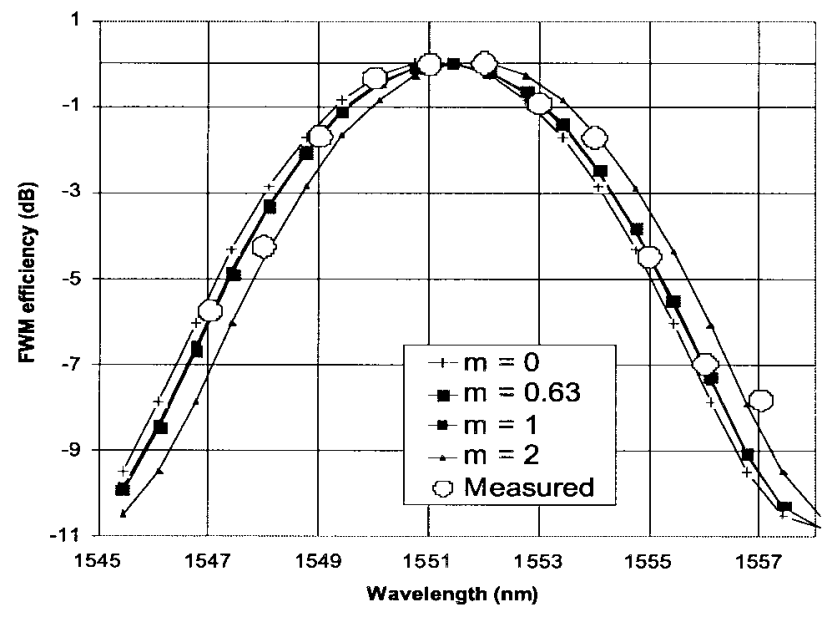

(a)

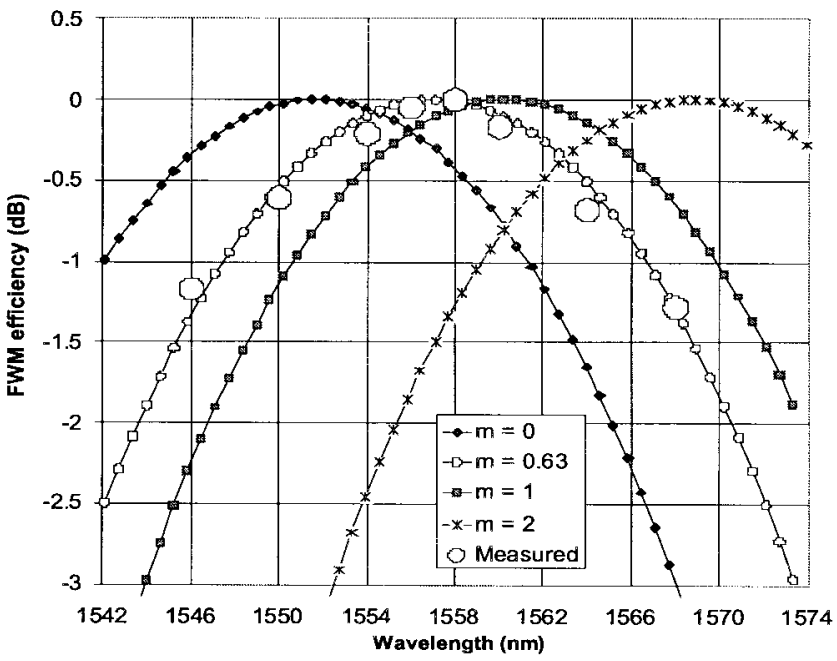

(b)

Fig. 3. FWM efficiency at different wavelengths (different fiber dispersion). Fiber length: $17.5 \mathrm{~km}$, loss: $0.25 \mathrm{~dB} / \mathrm{km}$, average zero-dispersion wavelength: $1551 \mathrm{~nm}$, dispersion slope: $0.075 \mathrm{ps} / \mathrm{km}-\mathrm{nm}$, fiber effective core area: 50 $\mu \mathrm{m}^{2}$, dispersion slope: $0.075 \mathrm{ps} / \mathrm{km}-\mathrm{nm}$. (a) $8 \mathrm{~mW}$ channel power and $1.0 \mathrm{~nm}$ channel spacing. (b) $40 \mathrm{~mW}$ channel power and $0.4 \mathrm{~nm}$ channel spacing.

only small differences are seen between the old and new phase-matching factor.

A different scenario is presented in Fig. 3(b). In this case, we increased the channel power to $40 \mathrm{~mW}(16 \mathrm{dBm})$ and decreased the channel spacing to $0.4 \mathrm{~nm}$. Here, the influence of SPM and XPM on FWM are significant and the peak of FWM has shifted several nanometers. The results calculated using the new phase-matching factor $(m=0.63)$ agree well with the measured data, whereas the older formulas exhibit significant errors. The linear phase-matching factor $(m=0)$, since it is unaffected by channel power, still predicts the location of peak FWM at the zero-dispersion wavelength, which is about $5 \mathrm{~nm}$ from the measured peak FWM. Fig. 3(b) also shows the calculated FWM efficiency using the phase-matching factors for $m=1$ and $m=2$. The calculated FWM peaks from the measured peak is about $3 \mathrm{~nm}$ away for $m=1$ and $12 \mathrm{~nm}$ away for $m=2$. Clearly, if FWM is used to measure the zerodispersion wavelength of DSF, the old formulas may result in significant errors.

\section{CONCLUSION}

In summary, we have shown that the influence of SPM and XPM on the FWM process becomes significant when the transmitted channel powers are large and the fiber dispersion or the channel spacing is small. Consequently, the conventional formula for calculating the phase-matching conditions for FWM produces significant errors by neglecting the SPM and XPM effects. We derived a new phase-matching factor by including these effects, resulting in an additional powerdependent term with very concise form. Both experimental and calculated results show that the new phase-matching factor produces greatly improved estimates of FWM power generation.

\section{REFERENCES}

[1] R. W. Tkach, A. R. Chraplyvy, F. Forghieri, A. H. Gnauck, and R. M. Derosier, "Four-photon mixing and high-speed WDM systems," $J$. Lightwave Technol., vol. 13, pp. 841-8849, May 1995.

[2] K. O. Hill, D. C. Johnson, B. S. Kawasaki, and R. I. MacDonald, "CW three-wave mixing in single-mode fibers," J. Appl. Phys., vol. 49, no. 10, pp. 5098-5106, Oct. 1978.

[3] N. Shibata, R. P. Braun, and R. G. Warrts, "Phase-mismatch dependence of efficiency of wave generation through four-wave mixing in a singlemode fiber," IEEE J. Quantum Electron., vol. QE-23, pp. 1205-1211, July 1987.

[4] F. Forghieri, R. W. Tkach, and A. R. Chraplyvy, "WDM system with unequally spaced channels," J. Lightwave Technol., vol. 13, pp. 889-897, May 1995.

[5] K. Inoue and H. Toba, "Fiber four-wave mixing in multi-amplifier systems with nonuniform chromatic dispersion," J. Lightwave Technol., vol. 13, pp. 89-93, Jan. 1995.

[6] W. Zeiler, F. D. Pasquale, P. Bayvl, and J. E. Midwinter, "Modeling of four-wave mixng and gain peaking in amplifid WDM optical communication systems and networks," J. Lightwave Technol., vol. 14, pp. 1933-1942, Sept. 1996.

[7] B. Hwang and O. K. Tonguz, "A generalized sub-optimum unequally spaced channel allocation techniques-Part I: In IM/DD WDM systems," IEEE Trans. Commun., vol. 46, pp. 1027-1037, Aug. 1998.

[8] G. P. Agrawal, Nonlinear Optics. San Diego, CA: Academic, 1995.

[9] J. A. Armstrong, N. Bloembergen, J. Ducuing, and P. S. Pershan, "Interaction between light waves in a nonlinear dielectric," Physic. Rev., vol. 127, no. 6, pp. 1918-1939, Sept. 1963.

[10] R. H. Stolen and J. E. Bjorkholm, "Parametric amplification and frequency conversion in optical fibers," IEEE J. Quantum Electron., vol. QE-18, pp. 1062-1071, July 1982.

[11] P. G. Agrawal, "Modulation instability induced by cross-phase modulation in optical fibers," Phys. Rev. A, vol. 39, no. 7, pp. 3406-3412, Apr. 1989.

[12] A. Vatarescu, "Light conversion in nonlinear monomode optical fibrs," J. Lightwave Technol., vol. LT-5, pp. 1652-1659, Dec. 1987.

[13] W. Huang and J. Hong, "A coupled-mode analysis of modulation instability in optical fibers," J. Lightwave Technol., vol. 10, pp. 156-62, Feb. 1992.

[14] B. S. Cavalcanti, J. C. Cressoni, H. R. Cruz, and A. S. Gouveia-Neto, "Modulation instability in the region og minimum group-VELOCITY dispersion of single-mode optical fibers via an extended nonlinear schrodinger equation," Physic. Rev. A, vol. 43, no. 11, pp. 6162-6165, June 1991.

[15] F. K. Abdullaev, S. A. Darmanyan, S. Bischoff, P. L. Christiansen, and M. P. Sorensen, "Modulation instability in optical fibers near the zero-dispersion point," Opt. Commun., vol. 108, pp. 60-64, May 15, 1994.

[16] M. Karlsson, "Modulation instability in lossy optical fibers," J. Optic. Soc. Amer. B, vol. 12, no. 11, pp. 2071-2077, Nov. 1995.

[17] P. O. Hedekvist, M. Karlsson, and P. A. Andrekson, "Fiber fourwave mixng demultiplexing with inherent parametric amplification," $J$. Lightwave Technol., vol. 15, no. 11, pp. 2501-2058, Nov. 1997.

[18] T. Yamamoto and M. Nakazawa, "Highly efficient four-wave mixing in an optical fiber with intensity dependent phase matching," IEEE Photon. Technol. Lett., vol. 9, pp. 327-329, Mar. 1997.

[19] D. Marcuse, A. R. Chraplyvy, and R. W. Tkach, "Effect of fiber nonlinearity on long-distance transmission," J. Lightwave Technol., vol. 9, no. 1, pp. 121-128, Jan. 1991. 


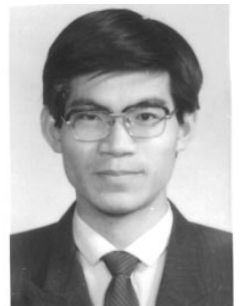

Shuxian Song (M'98) received the B.S. degree in electrical engineering from Shandong University in 1985 and the M.S. degree in electrical engineering from Beijing Institute of Technology in 1988 in the People's Republic of China. He received the Ph.D degree with Honors in electrical engineering from The University of Kansas, Lawrence, in 1998.

From 1988 to 1994 , he worked as a faculty member in Beijing Institute of Technology, China, where he was an Assistant Professor from 1988 to 1990 and a Lecturer from 1991 to 1994 . He worked as a Research Assistant in the Lightwave Systems Laboratory of the Information and Telecommunication Technology Center (ITTC) in The University of Kansas while pursuing the Ph.D degree. In 1998, he joined CIENA Corporation, Linthicum, MD, working on the modeling and design of wavelength division multiplexed (WDM) systems. Currently, he is in charge of the system level design on the next-generation WDM systems in CIENA. $\mathrm{He}$ has more than 30 journal papers and conference presentations. He is also the inventor or co-inventor for several inventions, of which one patent was awarded and three are pending

Dr. Song received the Paper Award of the Year 1991 given by the Chinese Electronics Institute (CIE) and the Young Scientist Paper Award given by the Bioelectromagnetic Society of China in 1992. He is currently is a member of LEOS, the IEEE Communications Society, and the Optical Society of America (OSA).

Christopher T. Allen (M'94-SM'95) was born in Independence, MO, on October 7, 1958. He received the B.S., M.S., and Ph.D. degrees in electrical engineering from The University of Kansas, Lawrence, in 1980, 1982, and 1984, respectively.

From 1984 to 1990, he was with Sandia National Laboratories, Albuquerque, NM, working in exploratory radar systems and development of high-speed digital systems. From 1990 to 1994, he was with the Allied Signal Kansas City Division, Kansas City, MO, where he worked in the areas of high-speed digital design, radar systems analysis, and multichip module development. Since August 1994, he has been a faculty member in the Electrical Engineering and Computer Science Department at The University of Kansas, Lawrence. His research interests include high-speed digital circuits, microwave remote sensing, radar systems, and photonics/lightwave technologies.

Dr. Allen has served as a technical reviewer for various IEEE journals and Remote Sensing of the Environment, Geophysics-The Journal of the Society of Exploration Geophysicists, and the Journal of Glaciology. He is a member of Phi Kappa Phi, Tau Beta Pi, Eta Kappa Nu, and the International Union of Radio Science (URSI).
Kenneth R. Demarest (S'78-M'79-SM'99) was born in Hackensack, NJ, on December 16, 1952. He received the B.S. degree in electrical engineering from John Brown University, Siloam Springs, AR, in 1974, and the M.S. and Ph.D. degrees in electrical engineering from The Ohio State University, Columbus, in 1976 and 1980, respectively.

From 1974 to 1979, he was a Graduate Research Associate with the ElectroScience Laboratory at The Ohio State University. From 1979 to 1984 he was an Assistant Professor in the electrical engineering department of Lafayette College, Easton, PA. Since 1984, he has been with the electrical engineering and computer science department at the University of Kansas, Lawrence, most recently as a Professor. His research interests are in the areas of fiber-optic communications and electromagnetics. He is the author of a number of papers, book chapters, and the book Engineering Electromagnetics (New York: Prentice-Hall, 1998).

Dr. Demarest is a member Eta Kappa $\mathrm{Nu}$ and the International Union of Radio Science Commission B.

Rongqing Hui (A'94-M'97-SM'97) received the B.S. degree in microwave communications in 1989 and the M.S. degree in lightwave technology in 1988, both from Beijing University of Posts \& Telecommunications, Beijing, China. He received the $\mathrm{Ph} . \mathrm{D}$ degree in electrical engineering from Politecnico di Torino, Torino, Italy, in 1993.

From 1982 to 1985, he taught at the Physics Department of Anhui University, Hefei, China, where he also conducted research on optical fibers and fiber sensors. From 1985 to 1989, he was with the Optical Communication Laboratory of Beijing University of Posts \& Telecommunications, where he worked in coherent optical fiber communication systems and components. From 1989 to 1990, he held a research fellowship from the Fundazione Ugo Bordoni, Rome, Italy, working on nonlinear effects and optical injection locking of semiconductor laser devices. From 1990 to 1993, he was with the Department of Electronics, Politecnico di Torino, where he worked on optical communications and single frequency semiconductor laser devices. During this period, he also held a fellowship from the Italian Telecommunication research Center (CSELT), Torino, Italy, where his research subject was polarization-insensitive coherent optical communication systems. He spent one year, from 1993 to 1994, as a postdoctoral research fellow working on optical systems and networks architecture at the University of Ottawa, Ont., Canada. He joined Bell-Northern Research (now part of Nortel), Ottawa, Ont., Canada, in 1994 as a Member of Scientific Staff, where he has worked in the research and development of high-speed optical transport networks. Since September 1997, he has been a faculty member in the Electrical Engineering and Computer Science Department, The University of Kansas. $\mathrm{He}$ has published more than 40 technique papers as an author or coauthor in leading engineering journals, in addition to numerous papers presented in internal conferences. He also acted as a technique reviewer for various IEEE, IEE, and OSA journals. 